zero and one, are "stubborn"; the 'abundant' number 18 , whose divisors' sum is greater than itself, "secretly carried a heavy burden"; the 'deficient' number 14, whose divisors' sum is less, "fell mute in the face of its terrible lack". These tactics help readers to understand the concepts in a creative way, although not everyone will want to follow the strings of equations and diagrams that occasionally invade the prose.

Mathematics inspires a reverence in the Professor. There are repeated references to numbers as mystical entities, existing on the pages of "God's notebook". And in a world that is constantly being whisked out from underfoot, the Professor is comforted by the predictability of things he can remember: mathematics, of course, but also statistics on baseball cards, or how to predict the position of Venus in the evening sky. A single mother with a dead-end job, the Housekeeper also finds maths a source of clarity: "I needed the sense that this invisible world was somehow propping up the visible one, that this one, true line extended infinitely, without width or area, confidently piercing through the shadows. Somehow, this line would help me find peace."

The mathematical lessons weave through a narrative that is uneventful but pervaded

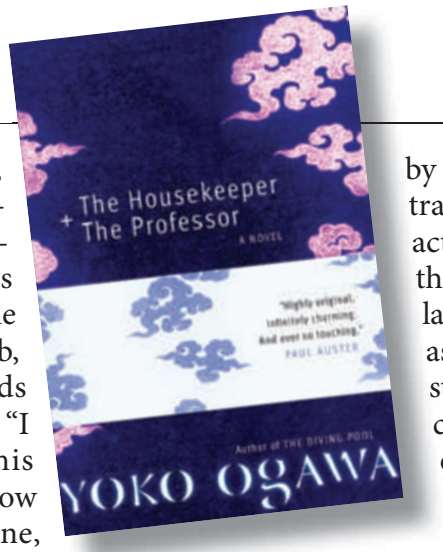

by a sense of beauty. Although the translation is fresh, and the characters could be of any nationality, there is a Japanese ambience. The language is as precise and graceful as a tea ceremony, and the backstory as sparsely sketched as shodo calligraphy. And if the Housekeeper's newly kindled enthusiasm for numbers sometimes stretches the reader's credulity, the book is so charming that the author is forgiven.

Jennifer Rohn is a cell biologist at University College London, London WC1E 6BT, UK, editor of www.LabLit.com and author of the novel Experimental Heart.

e-mail: jenny@lablit.com

\title{
Reflecting the impossible
}

\author{
Virtual Worlds: M. C. Escher and Paradox \\ Portland Art Museum, Portland, Oregon \\ Until 13 September
}

Two artists embody the saying that mathematics and art are so far apart they are practically neighbours - Leonardo da Vinci (1452-1519) and Maurits Cornelius Escher (1898-1972). Whereas Da Vinci searched for the possible, generating functional designs such as his flying machines, Escher searched for the impossible, creating images by distorting nature's

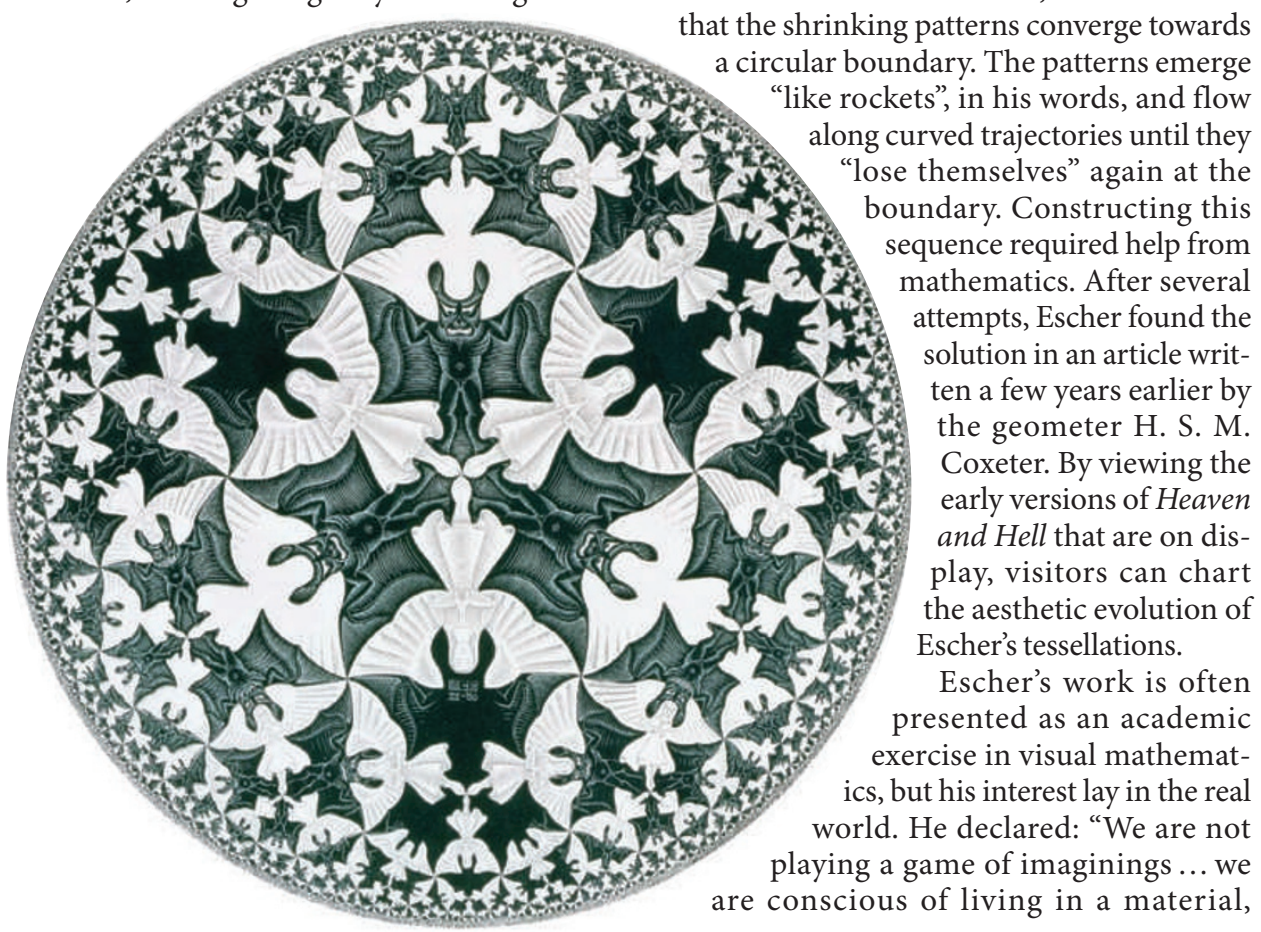

rules. Escher's techniques are explored in the retrospective exhibition Virtual Worlds: M. C. Escher and Paradox, now showing at the Portland Art Museum in Oregon.

Escher's prints of tessellations form the heart of the show. Heaven and Hell (1960; pictured) is the most intricate, incorporating patterns that repeat at many size scales, inspired by the tile designs of the Alhambra palace in Granada, Spain. The print's alternative name, Circle Limit IV, reflects the mathematical challenge that the artist undertook to make it.

To achieve visual balance, Escher insisted that the shrinking patterns converge towards cular boundary. The patterns emerge "ke rockets", in his words, and flow "lose themselves" again at the undary. Constructing this mathematics. After several attempts, Escher found the solution in an article written a few years earlier by the geometer H. S. M. Coxeter. By viewing the early versions of Heaven and Hell that are on disthe aesthetic evolution of Escher's tessellations

Escher's work is often presented as an academic exercise in visual mathematics, but his interest lay in the real playing a game of imaginings ... we are conscious of living in a material, play, visitors can chart three-dimensional reality." This is emphasized in his tree sketches, which show how the patterns of branches repeat at different scales and distort when reflected in the rippled surface of a pond.

The patterns in Heaven and Hell do not replicate those found in nature - their scalings shrink at a different rate. Nature produces fractal structures, as shown in one exhibit comprising a spherical mirror positioned at the centre of a cube of mirrors. Coloured light rays bounce around the mirrors, their many reflections setting up a fractal pattern when viewed from outside. But Escher did not depict this geometry, instead using the hyperbolic one described in Coxeter's article.

Intriguingly, Heaven and Hell was created many years before Benoît Mandelbrot's book The Fractal Geometry of Nature (W. H. Freeman, 1982), which made nature's scaling properties well known. The exhibition thus asks to what extent Escher knew about these natural rules. The artist hints that he was conscious of them and chose alternatives: "The reality around us ... is too common, too dull, too ordinary for us. We hanker after the unnatural or supernatural, that which does not exist, a miracle." Perhaps he achieved this miracle in what he referred to as the "deep, deep infinity" of his repeating patterns.

Richard Taylor is associate professor of physics, psychology and art in the Department of Physics at the University of Oregon, Eugene, Oregon 97403, USA

e-mail:rpt@uoregon.edu

\section{Correction}

The book review 'Cooking debate goes off the boil' by Pat Shipman (Nature 459, 1059-1060; 2009) incorrectly stated that carbohydrates can be obtained from fat and marrow when, in fact, it is fat that is obtained from these sources. 